\title{
Using volume shifts to improve the description of speed of sound and other derivative properties with cubic Equations of State.
}

\author{
André M. Palma ${ }^{1}$, António J. Queimada ${ }^{2, *}$ and João A. P. Coutinho ${ }^{1}$ \\ ${ }^{1}$ CICECO, Chemistry Department, University of Aveiro, Campus de Santiago, 3810-193 Aveiro, \\ Portugal. \\ ${ }^{2}$ KBC Advanced Technologies Limited (A Yokogawa Company), 42-50 Hersham Road, Walton- \\ on-Thames, Surrey, United Kingdom, KT12 1RZ. \\ *Corresponding author. E-mail address: aqueimada@kbcat.com
}

Supplementary Data 
Table A1 - Pure compound parameters using the modified EoS parameterizations.

\begin{tabular}{|c|c|c|c|c|c|c|c|c|c|}
\hline Compound & $\begin{array}{c}a_{c} \\
\left(\mathbf{P a}^{6} \mathbf{m}^{6} \cdot \mathrm{mol}^{-2}\right)\end{array}$ & $\begin{array}{c}b \cdot 10^{5} \\
\left(\mathbf{m}^{3} \cdot \mathrm{mol}^{-1}\right)\end{array}$ & $\mathbf{c}_{1}$ & $\mathbf{c}_{2}$ & $\mathbf{c}_{3}$ & $\mathbf{c}_{4}$ & $\mathbf{c}_{5}$ & $\beta .10^{2}$ & $\varepsilon\left(\mathrm{J} \cdot \mathrm{mol}^{-1}\right)$ \\
\hline $\begin{array}{l}\text { hexane } \\
\text { (SRK) }\end{array}$ & 2.53 & 12.12 & 1.06 & -2.01 & 12.02 & -33.1 & 35.1 & & \\
\hline $\begin{array}{l}\text { hexane } \\
\text { (PR) }\end{array}$ & 2.70 & 10.88 & 0.88 & -1.38 & 9.61 & -27.6 & 29.9 & & \\
\hline $\begin{array}{l}\text { decane } \\
\text { (SRK) }\end{array}$ & 5.32 & 21.00 & 1.41 & -3.73 & 28.05 & -102.9 & 148.3 & & \\
\hline $\begin{array}{l}\text { decane } \\
\text { (PR) }\end{array}$ & 5.69 & 18.85 & 1.20 & -2.77 & 22.89 & -85.7 & 124.3 & & \\
\hline $\begin{array}{l}\text { hexadecane } \\
\text { (SRK) }\end{array}$ & 10.93 & 36.89 & 1.90 & -6.28 & 43.88 & -144.7 & 182.8 & & \\
\hline $\begin{array}{l}\text { benzene } \\
\text { (SRK) }\end{array}$ & 1.91 & 8.27 & 0.91 & -1.94 & 13.14 & -41.1 & 50.8 & & \\
\hline $\begin{array}{l}\text { benzene } \\
\text { (PR) }\end{array}$ & 2.04 & 7.42 & 0.74 & -1.36 & 10.79 & -34.93 & 43.72 & & \\
\hline $\begin{array}{l}\text { toluene } \\
\text { (SRK) }\end{array}$ & 2.52 & 10.39 & 1.01 & -2.11 & 14.07 & -45.4 & 57.1 & & \\
\hline $\begin{array}{l}\text { toluene } \\
\text { (PR) }\end{array}$ & 2.70 & 9.33 & 0.83 & -1.47 & 11.41 & -38.2 & 48.9 & & \\
\hline methanol & 0.68 & 4.61 & 0.90 & -2.47 & 3.26 & 0.0 & 0.0 & 0.465 & 24913 \\
\hline ethanol & 1.13 & 6.40 & 1.04 & -1.46 & -0.79 & 3.8 & 0.0 & 0.165 & 24913 \\
\hline 1-butanol & 2.07 & 9.45 & 1.13 & -1.38 & 8.04 & -31.5 & 37.8 & 0.065 & 24913 \\
\hline 1-octanol & 4.48 & 17.17 & 1.63 & -6.76 & 35.8 & -81.8 & 67.7 & 0.065 & 24913 \\
\hline $\begin{array}{c}\text { ethylene } \\
\text { glycol }\end{array}$ & 1.72 & 6.87 & 1.10 & -3.64 & 12.16 & -37.1 & 42.5 & 0.162 & 24913 \\
\hline $\begin{array}{c}1,3- \\
\text { propanediol }\end{array}$ & 2.25 & 8.27 & 1.37 & -2.50 & 6.27 & -23.1 & 28.4 & 0.075 & 24913 \\
\hline water & 0.43 & 2.39 & 0.56 & -2.54 & -2.01 & 1.5 & 8.6 & 0.483 & 22013 \\
\hline
\end{tabular}


Table A2 - Information on the volume shifts fitted for each compound. When fitted to density the volume shift was always determined at $0.7 T_{r}$.

\begin{tabular}{|c|c|c|c|c|}
\hline \multirow{2}{*}{ Compound } & \multirow{2}{*}{ equation } & \multicolumn{2}{|c|}{ volume shift $\left(\mathrm{dm}^{3} \cdot \mathrm{kmol}^{-1}\right)$} & \multirow{2}{*}{$\begin{array}{c}T \text { of fitting (K) } \\
\text { fit speed of sound }\end{array}$} \\
\hline & & fit density & fit speed of sound & \\
\hline water & CPA & 11.6 & 2.9 & 383 \\
\hline methanol & CPA & 18.6 & 6.7 & 298 \\
\hline ethanol & CPA & 19.1 & 5.8 & 293 \\
\hline 1-butanol & CPA & 17.0 & 5.2 & 318 \\
\hline 1-octanol & CPA & 28.9 & 9.6 & 313 \\
\hline ethylene glycol & CPA & 20.4 & 1.5 & 293 \\
\hline 1,3-propanediol & CPA & 18.8 & 11.5 & 330 \\
\hline n-hexane & SRK & 19.4 & -38.8 & 323 \\
\hline n-hexane & PR & 0.6 & -29.9 & 323 \\
\hline n-decane & SRK & 47.8 & -28.0 & 313 \\
\hline n-decane & PR & 16.3 & -10.0 & 313 \\
\hline n-hexadecane & SRK & 116.9 & 3.0 & 328 \\
\hline benzene & SRK & 14.1 & -23.9 & 323 \\
\hline benzene & PR & 0.4 & -17.9 & 323 \\
\hline toluene & SRK & 18.6 & -22.3 & 323 \\
\hline toluene & PR & 2.3 & -14.1 & 323 \\
\hline
\end{tabular}



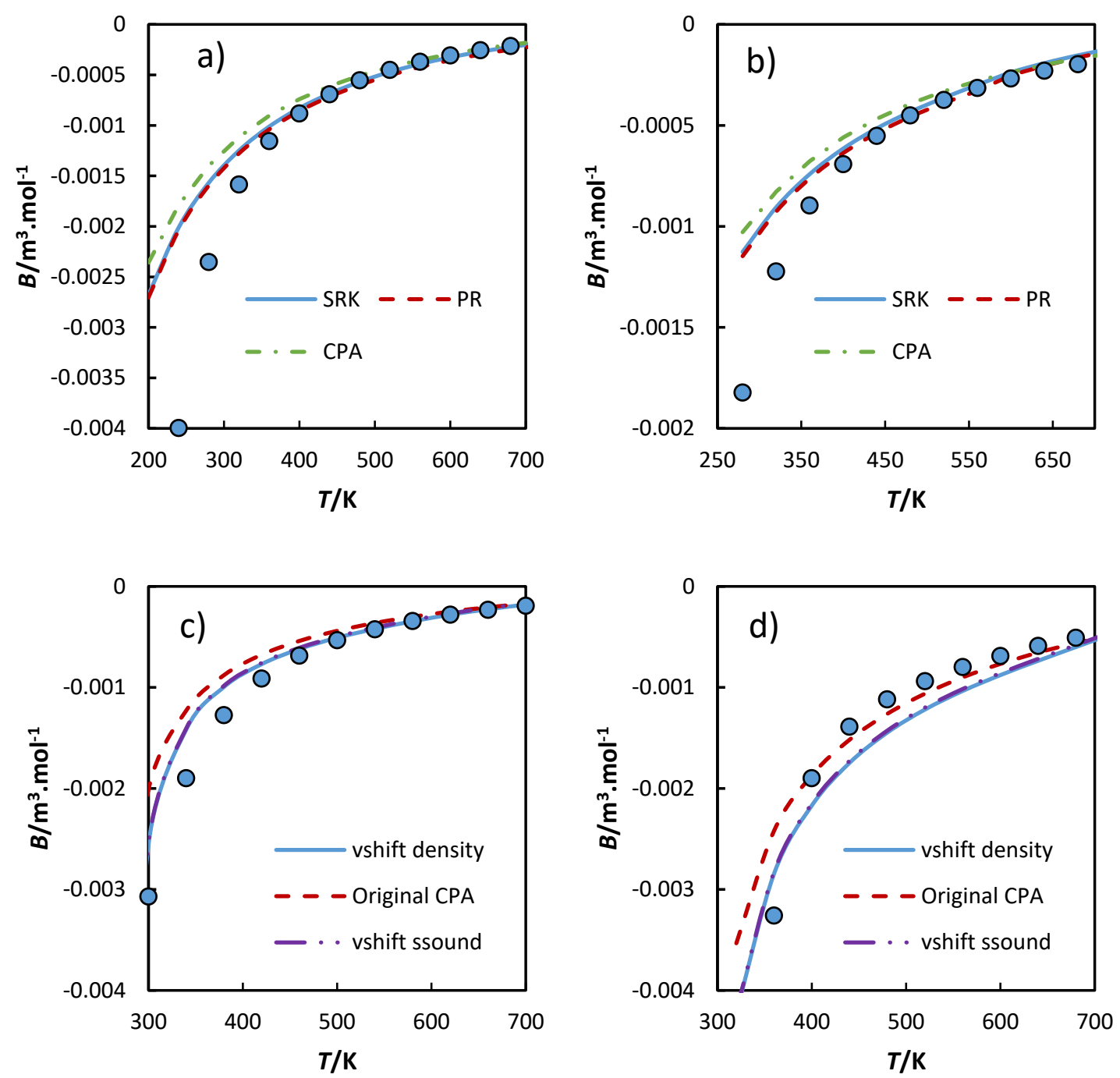

Figure A1 - Second virial coefficient for hexane (a), benzene (b), 1-butanol (c) and 1-octanol (d). CPA parameters are from Oliveira et al. ${ }^{1}$ Data taken from the correlations present on Multiflash. ${ }^{2}$ 

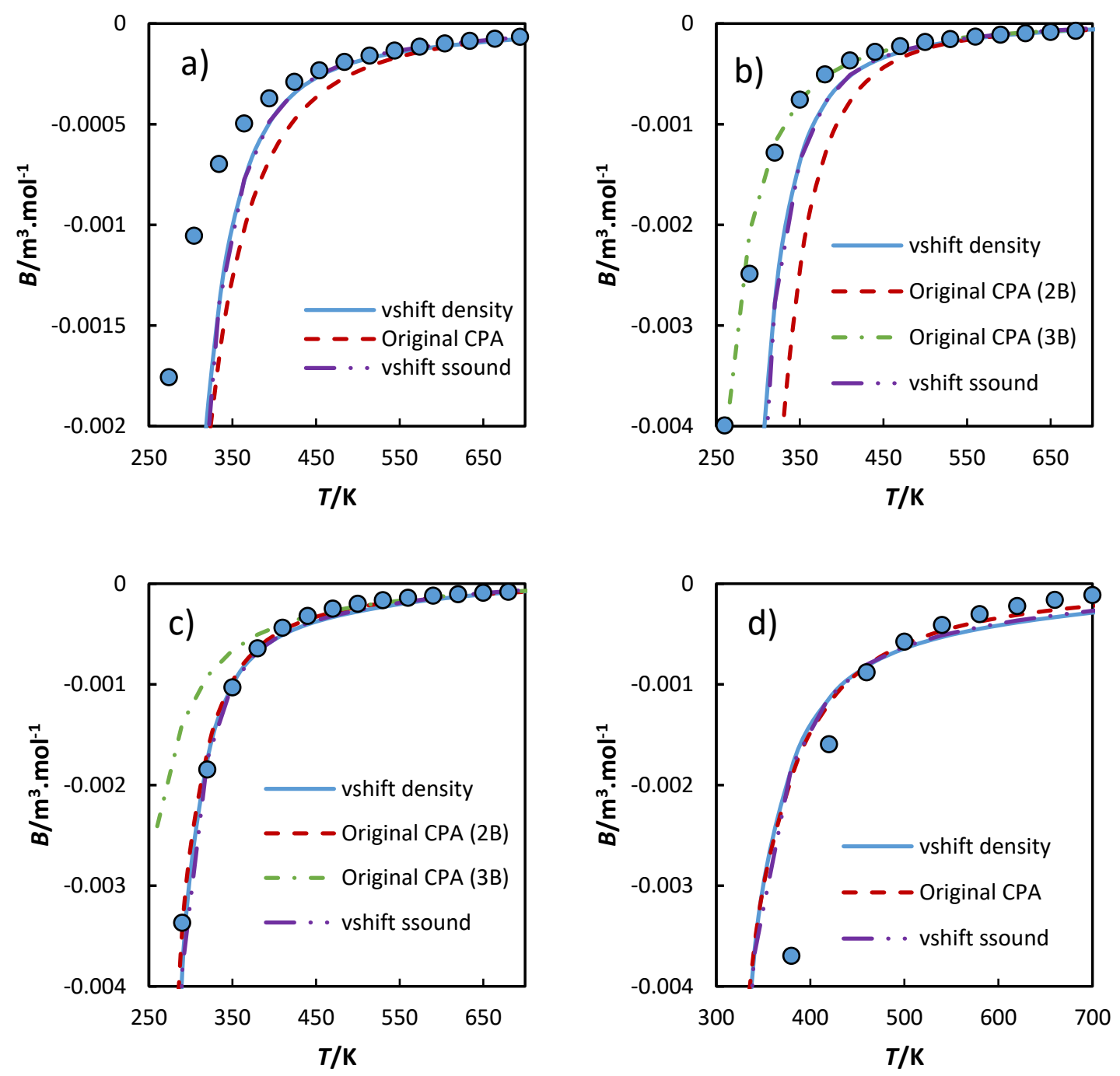

Figure A2 - Second virial coefficient for a) water, b) methanol, c) ethanol and d) ethylene glycol. Original CPA parameters taken from a CPA review. ${ }^{3}$ Data taken from the correlations of Multiflash. ${ }^{2}$

\section{References}

(1) Oliveira, M. B.; Marrucho, I. M.; Coutinho, J. A. P.; Queimada, A. J. Surface Tensions of Chain Molecules through a Combination of the Gradient Theory with the CPA EoS. Fluid Phase Equilib. 2008, 267 (1), 83-91. https://doi.org/10.1016/j.fluid.2011.01.015.

(2) MULTIFLASH Version 6.1, KBC Process Technology, London, United Kingdom.

(3) Kontogeorgis, G. M.; Michelsen, M. L.; Folas, G. K.; Derawi, S.; Von Solms, N.; Stenby, E. $\mathrm{H}$. Ten Years with the CPA (Cubic-Plus-Association) Equation of State. Part 1. Pure Compounds and Self-Associating Systems. Ind. Eng. Chem. Res. 2006, 45 (14), 4855-4868. https://doi.org/10.1021/ie051305v. 